\title{
Sensitivity to Gamma-Ray Bursts of a Nanosatellite MeV Telescope with a Silicon Tracker
}

\author{
Riccardo Rando $^{1,2}$ (10), Sofia Canevarolo ${ }^{1}$, Hubing Xiao ${ }^{1,2,3}$ (1) , and Denis Bastieri ${ }^{1,2,3}$ (1) \\ ${ }^{1}$ Dipartimento di Fisica \& Astronomia “G. Galilei," Università di Padova, I-35131 Padova, Italy; riccardo.rando@unipd.it \\ ${ }^{2}$ Istituto Nazionale di Fisica Nucleare, Sezione di Padova, I-35131 Padova, Italy \\ ${ }^{3}$ Center for Astrophysics, Guangzhou University, CN-510006 Guangzhou, People's Republic of China \\ Received 2018 December 14; revised 2019 May 20; accepted 2019 May 22; published 2019 July 2
}

\begin{abstract}
We propose a nanosatellite Compton telescope based on a silicon tracker, both as a fast and low-cost mission for astrophysics in the $\mathrm{MeV}$ regime and as a pathfinder for future large-scale Compton orbital observatories. In this paper we assess the sensitivity of such an instrument for the observation of gamma-ray bursts.
\end{abstract}

Key words: astroparticle physics - gamma-ray burst: general - gamma rays: general - instrumentation: detectors

\section{Introduction}

Since the deorbiting of the Compton Gamma-ray Observatory satellite, with the Compton Telescope (COMPTEL) onboard (Schönfelder et al. 1993), the region of the electromagnetic spectrum around $\sim 1 \mathrm{MeV}$ has been covered with significantly less sensitivity than other regions at higher and lower energies. New large-scale satellite observatories are being proposed, e.g., All-sky Medium Energy Gamma-ray Observatory (AMEGO; Moiseev et al. 2017) and enhanced ASTROGAM (De Angelis et al. 2017); these are characterized by a huge scientific return (De Angelis et al. 2018), high costs (several hundreds of millions, euros or dollars), and long development timescales $(\approx 10 \mathrm{yr})$.

We proposed a $\sim 1$ liter nanosatellite instrument as a pathfinder, based on the same Si tracker technology, arguing that it is also a valuable scientific instrument on its own account (Lucchetta et al. 2017). Remarkably, nanoscale satellites are being proposed as valuable detectors of gamma-ray bursts (GRBs) in the X-ray and gamma-ray bands, see, e.g., Chattopadhyay et al. (2018), Perkins et al. (2018), and Fiore et al. (2018), among many others. The opportunity to establish a network of many different experiments is also being investigated, ${ }^{4}$ since it improves on the capabilities of any single detector in several aspects.

In this paper we present an update on the design in Lucchetta et al. (2017) and estimate its performance for the observation of GRBs.

\section{The Instrument}

With respect to the preliminary instrument described in Lucchetta et al. (2017) we simplified the calorimeter design, making the bottom calorimeter thinner and replicating the design on the four sides, as shown in Figure 1. The main reason for this simplification stems from the fact that the detector was poorly performing in the pair-production regime, so we opted to design a purely Compton detector.

The readout system of the CsI crystals has been reviewed and optimized using Silicon photomultipliers and we relaxed the constraints on the spatial resolution of the tracker, based on Silicon-strip detector technology (SSD) down to $500 \mu \mathrm{m}$.

A plastic anticoincidence surrounds the detector to veto charged particles, and, as in the previous paper, no readout

\footnotetext{
4 https://asd.gsfc.nasa.gov/conferences/grb_nanosats/
}

electronics or support materials are yet included into the design. In particular, we have not yet allocated any space for the readout of the SSD. Either we will use commercially available space-validated double-sided SSDs and ASICs, making space for the latter in the design at the expense of effective area and sensitivity. More ambitiously, we can adapt existing active pixel technologies already employed in high-energy physics (Wang et al. 2017) to the requirements of operation in space. On this topic, the Italian National Institute for Nuclear Physics (INFN) approved the Advanced Readout CMOS Architectures with Depleted Integrated sensor Arrays (ARCADIA) project for the development of a fully depleted, low-power complementary metal-oxide semiconductor (CMOS) sensor design. Starting in 2019 January, it includes among its goals the portability to space applications, especially in terms of power consumption. If the design will have to be modified to make space for conventional readout electronics, there is a risk that the effective area and sensitivity will be affected significantly, see Section 5.

A summary of the current instrument design is in Table 1 . The satellite is assumed to be operating in a quasi-equatorial low-Earth orbit, pointing at the zenith.

The instrument we simulate is slightly wider than 1U (lateral dimensions are $11.5 \mathrm{~cm}$ instead of 10) for a volume of 1.32 liters. The mass of the active sensors is $3.2 \mathrm{~kg}$, most of it in the calorimeter. This is more than the cubesat standard $(1.33 \mathrm{~kg}$ per $1 \mathrm{U}$ ) but we need more space for all the satellite subsystems. As a baseline we consider a $6 \mathrm{U}$ satellite, with a volume and mass envelope of $8 \mathrm{~kg}$ to be shared by the scientific payload and all other subsystems. Additional passive material within the scientific payload, for structural elements and electronics, does not add much to the previous amount but causes scattering, affecting the event reconstruction. The instrument has 8944 readout channels (8640 in the tracker); assuming $0.3 \mathrm{~mW} /$ channel this translates into $2.7 \mathrm{~W}$ of power for the readout electronics, comparable with the requirements for attitude control and data transfer. This is manageable in a $6 \mathrm{U}$ satellite by passive cooling, but the heat from the readout electronics needs to be collected efficiently.

\section{Sensitivity to Steady Point Sources}

The sensitivity to steady point sources in the Compton regime in Lucchetta et al. (2017) is not significantly affected by 


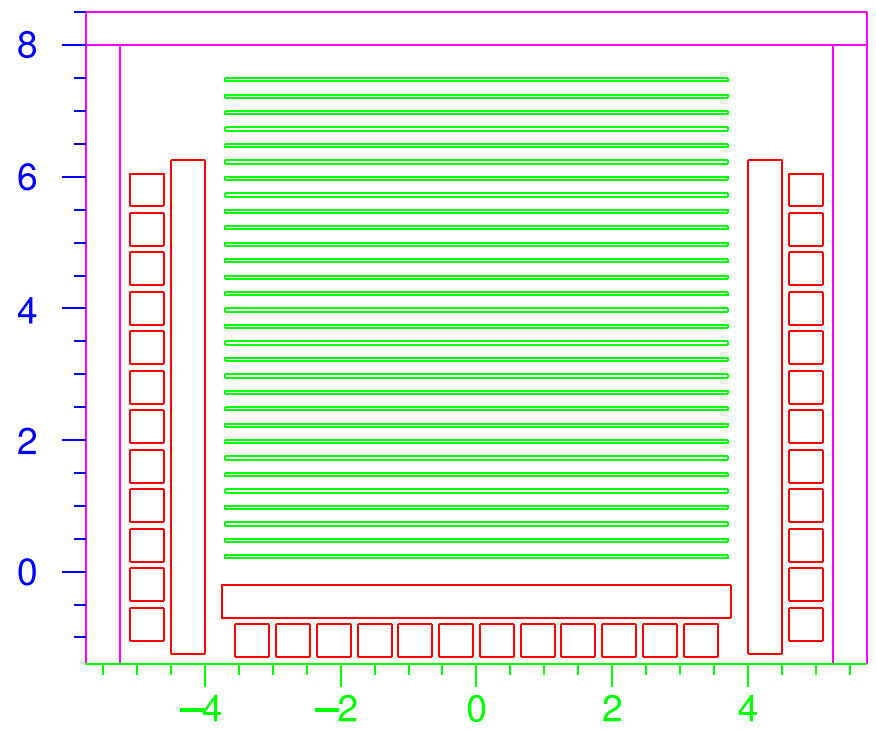

Figure 1. Active detectors of the MeV cubesat. The Si tracker is shown in green, the Cesium Iodine calorimeter in red, and the anticoincidence detector in magenta. For clarity we have removed the front and back calorimeter modules; The scales are in centimeters.

Table 1

Design Specifications

\begin{tabular}{ll}
\hline \hline Parameter & Value or Range \\
\hline Si tracker: layer area & $7.4 \times 7.4 \mathrm{~cm}^{2}$ \\
Si tracker: layer thickness & $500 \mu \mathrm{m}$ \\
Si tracker: number of layers & 30 \\
Si tracker: strip pitch & $500 \mu \mathrm{m}$ \\
Calorimeter: crystal dimensions & $0.5 \times 0.5 \times 7.5 \mathrm{~cm}^{3}$ \\
Calorimeter: depth resolution $(1 \sigma)$ & $0.5 \mathrm{~cm}$ \\
ACD: thickness & $0.5 \mathrm{~cm}$ \\
\hline
\end{tabular}

the change in design, nevertheless we re-estimate it with a slightly improved approach, leveraging once again the Megalib toolkit (Zoglauer et al. 2008). Following the basic guidelines defined in the previous paper, we distinguish events where the electron track is observed and the scattering plane is determined (tracked events) from all others (untracked). The main improvement of the analysis is the evaluation of the backgrounds. We still consider the same two sources of background photons: the photons produced by the interaction of primary protons with the atmosphere and the isotropic photon background, composed by unresolved gamma-ray sources and by the true extragalactic isotropic emission. The level of residual contamination by charged particles passing the anticoincidence shield is negligible in comparison in the recommended quasi-equatorial low-Earth orbit. The source is assumed to be at high Galactic latitude, and the Galactic plane is not simulated. In our previous paper, we evaluated the gamma-ray background event rates within a solid angle corresponding to the angular resolution analytically, relying on the source intensities and the instrument effective area. In this analysis, instead, we simulate the background photon sources $\left(10^{5} \mathrm{~s}\right)$ and we compute the background rate by counting how many events intersect the location of a candidate point source at a given inclination, taking into account the instrument's angular resolution as a function of energy and inclination (ARM, angular resolution measure, ARM, for all events, and scatter plane deviation, SPD, for tracked events).
The MegaLib toolkit used for simulation and analysis automatically rejects kinematically unlikely events; nonetheless many accepted events reconstruct to the wrong sky position due to incomplete absorption (one-third of the events at 1 $\mathrm{MeV}$ ), resulting in an additional quasi-isotropic background component. While this is accounted for in our simulation, the contribution from the Galactic plane is missing. We can estimate an upper limit by taking the Galactic plane emission in the inner Galaxy, integrated along latitude (Strong et al. 1994) and assuming it to be constant along the Galactic plane at all longitudes. From this we calculate that the Earth limb photons dominate by about one order of magnitude over this missing component.

In Figure 2, left, we show the $3 \sigma$ sensitivity for an observation of effective duration $t_{\text {eff }}=10^{6} \mathrm{~s}$, for all tracked events, and for the subset of events with energy deposition only in the bottom calorimeter assembly (tracked-bottom). In Figure 2, right, we show the signal-to-noise ratio for the same data selection. These two figures show that at the sensitivity limit the tracked-bottom events maintain a signal-to-noise ratio greater than $10 \%$ across the full energy range of the instrument, while suffering only a $30 \%$ decrease in sensitivity relative to all tracked events. A successful observation at a given signal-tonoise ratio (statistical error) implies that the systematic uncertainties are kept under control at the same level, and $10 \%$ can be taken as a reasonable goal based on the performance of existing instruments.

In the previous analysis we have assumed a point source at zenith, aligned with the boresight of the satellite. In Figure 3, left, we show the point source sensitivity, with the same conditions, as a function of the inclination of the incoming photon with respect to the instrument axis, for energy of the incoming photon in the band 562-1000 keV. No loss of field of view is evident until $90^{\circ}$, beyond that the bright Earth limb causes significant complications. The little dependence on the inclination angle means that the conversion of effective time into mission time is very close to the factor 2 caused by the observation of half of the sky at all times.

Events where the electron track is not observed (untracked) can be discarded in the case of the observation of relatively faint, steady sources, due to the poor signal-to-noise ratio $(<1 \%)$.

The nominal energy range for our detector is $100 \mathrm{keV}-$ $5 \mathrm{MeV}$, nonetheless we limit the analysis at $E<1.78 \mathrm{MeV}$ since beyond that point the amount of badly reconstructed events starts to become relevant, and some quality cuts must be applied. Such cuts depend strongly on the exact geometry and readout of the instrument so we postpone this time-consuming study until a definitive design is established.

\section{Sensitivity to GRBs}

We will assume that we can receive alerts, e.g., circulars of the Gamma-ray Coordinates Network, which provide us with the trigger time, the approximate length of the burst, and a rough estimate of the location and of the luminosity. In principle we could also generate some of these alerts internally, e.g., following the procedure in Narayana Bhat et al. (2016) and references within, but as we will see in this case we lose the advantage of a first location that benefits our analysis by limiting the region over which we sample the fluctuations of the background. 

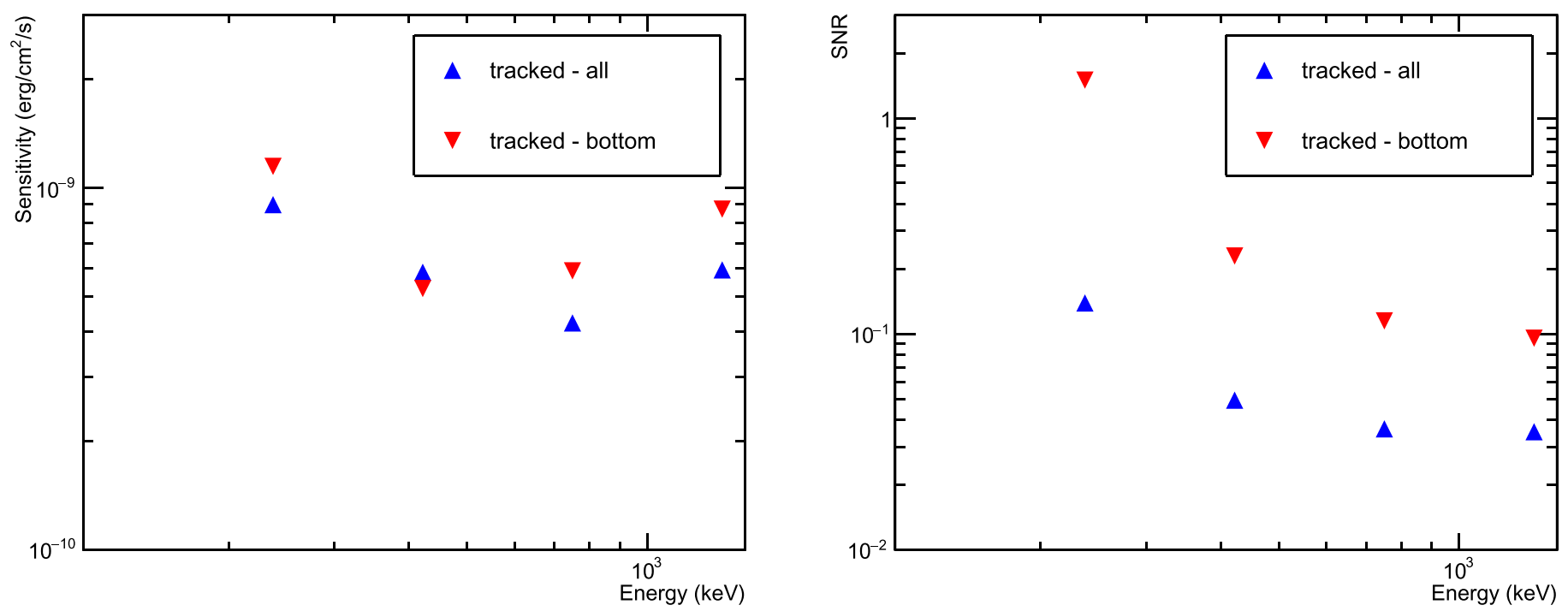

Figure 2. Sensitivity for a steady point source ( $3 \sigma$ and $T_{\text {eff }}=10^{6} \mathrm{~s}$, left) and signal-to-noise ratio (same $T_{\text {eff }}$ at the sensitivity limit, right) as a function of energy for an inclination of $\theta<14.5$.
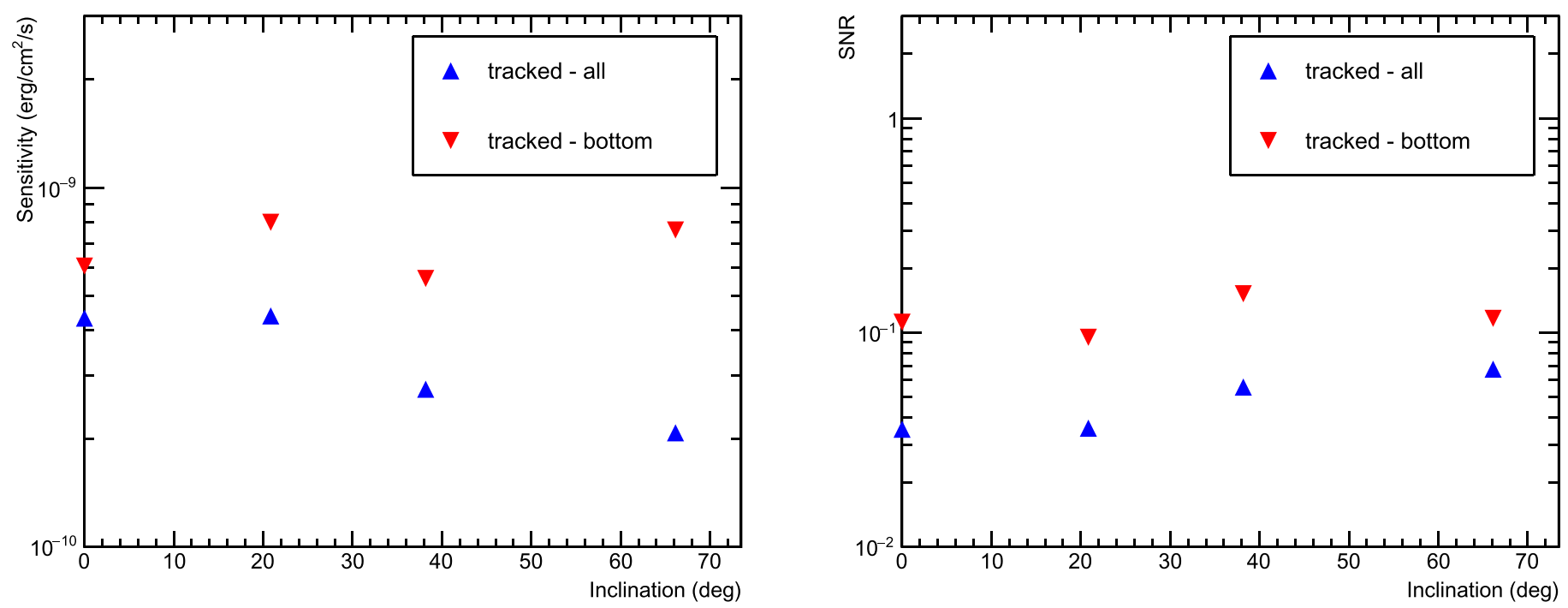

Figure 3. Sensitivity for a steady point source $\left(3 \sigma\right.$ and $T_{\text {eff }}=10^{6} \mathrm{~s}$, left) and signal-to-noise ratio (same $T_{\text {eff }}$ at the sensitivity limit, right) as a function of inclination from the axis for $562 \mathrm{keV}<E<1 \mathrm{MeV}$.

Our goal is to confirm the observation of the GRB with a simple, automated procedure and postpone localization, timing, and spectral analysis to a refined analysis later on, see, e.g., Cao et al. (2019). To claim detection we will require a signal $3 \sigma$ (pre-trials) above the background fluctuations, and at least nine detected photons. Since the number of trials is relatively low (less than 300 per year, based on the rate of GRBs in the FermiGBM catalog) we can keep the number of false positives below one per year of operation.

We proceed as follows.

1. A background simulation is performed, including secondary photons from Earth and extragalactic isotropic emission, as described in Lucchetta et al. (2017) and above. The simulated time interval is divided in time bands of increasing width, from $0.125 \mathrm{~s}$ to $64 \mathrm{~s}$. We create 2500 time slices for all time bands except the last one, where we have only 1560 .
2. For each slice in each band we do an imaging analysis (list mode likelihood; Zoglauer 2005), looking for the brightest spot in the sky. We use an extremely simplified model for the response of the detector: $1 \sigma$ Gaussian approximation for the cores of ARM and SPD, independent of energy and inclination. The parameters are obtained from the simulation of secondary photons from the Earth's atmosphere (ARM $1 \sigma$ is 6.7 for all events, SPD $1 \sigma$ is $34^{\circ} .2$ for tracked).

3 . For each time band we fill a histogram with the maximum values of the likelihood sky maps and we derive the $3 \sigma$ cutoff as the 0.997 th quantile. The cutoff values as a function of the duration of the time bands are then interpolated with a continuous function, allowing us to derive a cutoff value for each duration of a GRB.

4. We simulate all the GRBs in the Fermi-GBM catalog up to 2017 December 31 as point sources, using the preferred spectral model and the corresponding spectral 


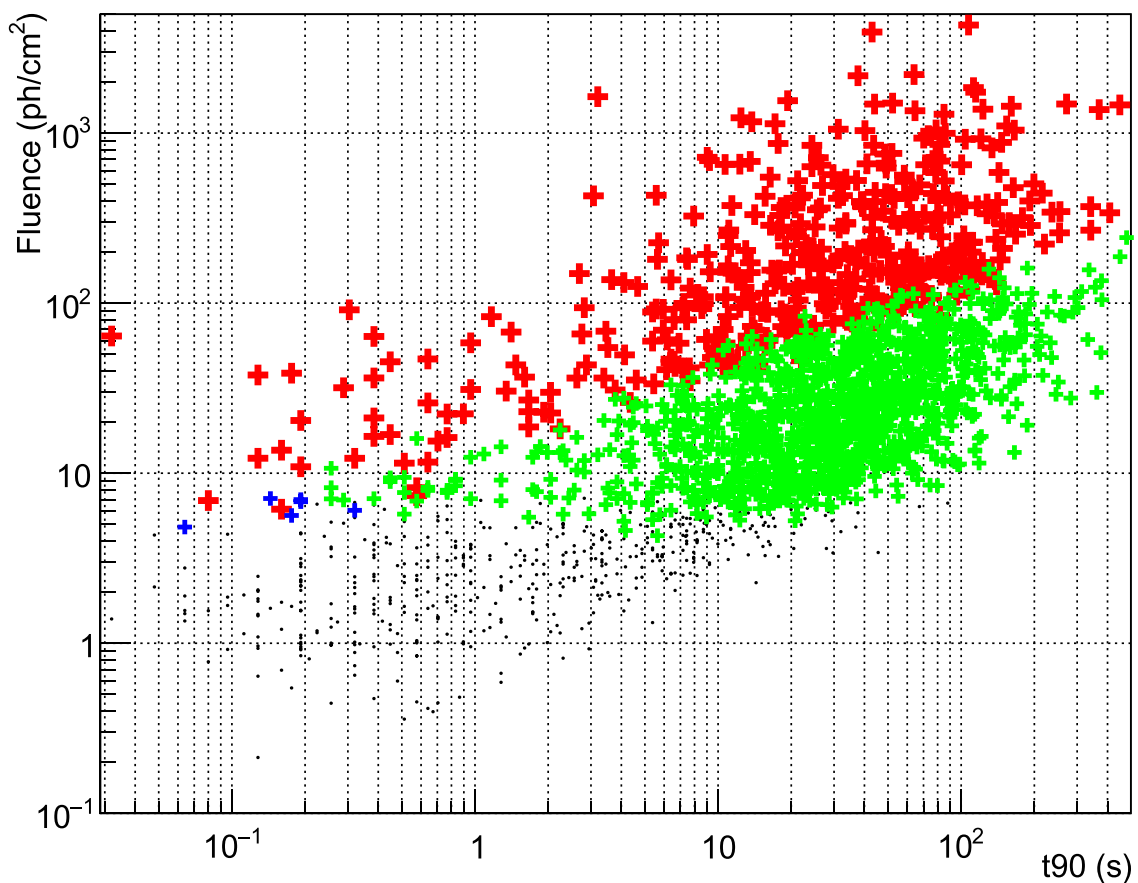

Figure 4. GRBs in the Fermi-GBM catalog, fluence $(100 \mathrm{keV}-5 \mathrm{MeV})$ vs. $t_{90}$. Red crosses: observable; blue crosses: passing the cutoff but less than nine photons; green crosses: at least nine photons but failing the cutoff; and dots: failing both requirements. Analysis: all events (tracked and untracked) for an initial location uncertainty of $15^{\circ}$, source at zenith. The analysis assumes an alert from an independent instrument providing coarse estimates of localization and flux.

parameters. The simulated source is set up to give $90 \%$ of the total GRB fluence in a time interval of length, $t_{90}$. In order to neglect the effect of statistical fluctuations in the model bursts, all GRB intensities are multiplied by a factor 5 .

5. For each GRB we perform the same imaging analysis we did for the background samples (using the same response model). We take the maximum value of likelihood in the skymap and we tally the number of detected photons. All values are divided by a factor 5 to compensate the scaling up in the previous step. GRBs with likelihood values above the background cutoff, evaluated for their $t_{90}$ and with at least nine detected photons, are considered as observed. We repeat this analysis by limiting the search range into a circular region of radius of $15^{\circ}$, with the center ranging from the local zenith down to inclination of $75^{\circ}$, to simulate the effect of searching inside a localization error circle from an alert from the Fermi-GBM.

The results, using all Compton events (tracked and untracked) and assuming an initial search window of $15^{\circ}$ centered at the zenith, are in Figure 4. The GRBs in the FermiGBM catalog are indicated by dots, and we plot the $90 \%$ fluence (in $\left.\mathrm{ph} \mathrm{cm}^{-2}\right)$ in the design energy band $(100 \mathrm{keV}-$ $5 \mathrm{MeV}$ ) versus the $t_{90}$ duration in seconds. Red, bold crosses indicate GRBs that we detect with the aforementioned procedure, blue crosses indicate bursts that are above the background cutoff but with less than nine collected photons, and green crosses indicate bursts with at least nine detected photons but failing the cutoff. The spectral shape of each GRB and of the background populations, convoluted with the energy dependence of the effective area of our instrument, cause the observed small overlap between the red, green, and blue populations.
The number of detected short GRBs (defined here as $t_{90}<2$ s) is $49 \pm 10$, to be compared with $630 \pm 220$ long GRBs. Taking into account the different field of view of our instrument (limited to half of the sky) and of the FermiGBM, we obtain a rate of $3.7 \pm 0.7$ short GRBs and $48 \pm 17$ long GRBs per year. ${ }^{5}$ The uncertainties above can be treated as maximum errors and include both statistical and systematic uncertainties, and are dominated by the evaluation of the background cutoff, in particular by the impact of statistical fluctuations in the simulated data set. In particular the uncertainties include the dependence on the inclination angle, as we verified by repeating the analysis for a similar search window centered at an inclination of $75^{\circ}$ from zenith, obtaining compatible results.

In case we do not have an initial search window, e.g., because the alert is generated internally, we have to search across the entire field of view and the background cutoff levels increase. Correspondingly, the number of expected short GRBs decreases by a factor $\sim 3$ suggesting that it may be not worth the effort to implement this functionality on board. The expected rate for long GRBs is less sensitive, dropping by a factor $\sim 2$.

Repeating the analysis using only tracked events we observe that the decrease in the cutoff values, which are mostly determined by the chance superposition of event arcs giving large likelihood values in the sky maps, is negligible in comparison to the loss of effective area. The number of detected GRBs falls dramatically to the point that only a few are detected (1-5) with the automated procedure described above.

\footnotetext{
5 The low cost of a nanosatellite mission suggests to place a second one in orbit with a phase difference of $180^{\circ}$, doubling the estimated rates and keeping the whole sky under surveillance at all times.
} 


\section{Conclusions}

We evaluated the performance as a GRB detector of a $\sim 1$ liter nanosatellite based on a Silicon tracker, and found that following an external alert with characteristics similar to those issued by the Fermi-GBM. we expect to detect about 4 short GRBs and about 50 long GRBs per year of mission with a rather simple automated procedure. It is conceivable that these figures can improve with a careful, dedicated analysis of interesting events, e.g., short GRBs associated to gravitationalwave events.

For relatively fainter, steady sources the sensitivity of the instrument is optimized by rejecting the intense backgrounds, mostly photons from the Earth's atmosphere. Under these conditions, the portion of the calorimeter surrounding the sides of the tracker is not used and only tracked events with the scattered gamma-ray hitting the bottom calorimeter are downlinked. On the contrary, for short observation times and relatively bright sources the performance is optimized by maximizing the effective area, therefore all events (including untracked ones) are used and the lateral calorimeter structure is necessary. Since GRBs are quite rare we will store all events in an internal buffer and transmit to the ground only the time segments surrounding burst alerts. We can appreciate the decrease in the event rate by looking at the background simulations, where we have about $7 \mathrm{~Hz}$ for all events, and $0.2 \mathrm{~Hz}$ for tracked-bottom events.

In conclusion such a small detector, even though not specifically designed for the detection of GRBs, can contribute precious data in the $\mathrm{MeV}$ energy band and be a worthy addition to the proposed network of nanosatellite GRB observatories.

A word of caution: to characterize the final detector we should take into account all details of the mechanical design, which will worsen the overall performance evaluated in the previous sections. In particular if we shrink the detector inside a 1 liter volume (1U), including the readout electronics, we estimate that the loss of effective area could be as much as a factor $\sim 3$, as inferred by comparison with previous similar instruments (e.g., Watanabe et al. 2014).

Software: MegaLib (Zoglauer et al. 2008).

\section{ORCID iDs}

Riccardo Rando (i) https://orcid.org/0000-0001-6992-818X

Hubing Xiao iㅛ https://orcid.org/0000-0001-8244-1229

Denis Bastieri iㅏ https://orcid.org/0000-0002-6954-8862

\section{References}

Cao, H., Bastieri, D., Rando, R., et al. 2019, ExA, 47, 129

Chattopadhyay, T., Falcon, A. D., Burrows, D. N., Fox, D. B., \& Palmer, D. 2018, Proc. SPIE, 10699, 106995S

De Angelis, A., Tatischeff, V., Tavani, M., et al. 2017, ExA, 44, 25

De Angelis, A., Tatischeff, V., Grenier, I. A., et al. 2018, JHEAp, 19, 1

Fiore, F., Burderi, L., Di Salvo, T., et al. 2018, Proc. SPIE, 10699, $106992 \mathrm{Q}$

Lucchetta, G., Berlato, F., Rando, R., et al. 2017, AJ, 153, 237

Moiseev, A. 2017, Proc. ICRC, 301, 798

Narayana Bhat, P., Meegan, C. A., von Kienlin, A., et al. 2016, ApJS, 223, 28

Perkins, J. S., Racusin, J., Briggs, M., et al. 2018, AAS Meeting , 231, 361.14

Schönfelder, V., Aarts, H., Bennett, K., et al. 1993, ApJS, 86, 657

Strong, A., Diehl, R., Schoenfelder, V., et al. 1994, ApJS, 92, 425

Wang, T., Rymaszewskia, P., Barbero, M., et al. 2017, JInst, 12, C01039

Watanabe, S., Tajima, H., Fukazawa, Y., et al. 2014, NIMPA, 765, 192

Zoglauer, A. 2005, PhD thesis, Max-Planck-Institut für Extraterrestrische Physik

Zoglauer, A., Andritschk, R., Boggset, S. E., et al. 2008, Proc. SPIE, 7011, $70113 \mathrm{~F}$ 